\title{
Editorial
}

\section{Desafíos de la pandemia COVID-19: Investigación en salud y su divulgación}

\section{Challenges of the COVID-19 pandemic: Health research and its dissemination}

En los inicios de la pandemia fue visible el desconocimiento sobre el impacto que tendría el COVID-19 en las distintas esferas de la sociedad, la crisis fue escalando y las repercusiones del COVID-19 siguen presentándose ${ }^{(1)}$ y se van descubriendo con los hallazgos de las investigaciones o la vigilancia epidemiológica lo va mostrando su impacto en la salud pública.

La investigación producida en América Latina y el Caribe (ALC) representa cerca del $4 \%$ del total de las publicaciones del mundo, y el mismo porcentaje en el área de Medicina y Ciencias de la Vida, solo un 1\% de las publicaciones sobre COVID-19 son de autores de instituciones basadas en la región ${ }^{(2)}$, y en el Perú la mayoría de la producción científica peruana se basa en análisis de fuentes secundarias, con escasos estudios experimentales ${ }^{(3)}$.

En un contexto donde $\mathrm{ALC}$ ha sido una de las regiones más afectadas durante la pandemia, aun así algunos países han destinado recursos adicionales para proyectos de investigación en ciencias (incluidas las sociales), y para el desarrollo tecnológico y de equipos médicos y fármacos, con el anhelo de poner a sus países en mejores condiciones para responder a esta epidemia y evitar depender de desarrollos extranjeros $^{(4)}$, por ello el compartir la investigación sobre la COVID-19 es esencial.

Hace algunos días se realizó el Primer congreso sobre COVID-19, organizada por el Instituto Nacional de Salud (INS) del Ministerio de Salud (MINSA), el Consejo Nacional de Ciencia, Tecnología e Innovación Tecnológica (CONCYTEC), el Instituto de Evaluación de Tecnologías en Salud e Investigación (IETSI) del Seguro Social del Perú (EsSalud), la Universidad Continental y la Secretaría de Gobierno y Transformación Digital de la Presidencia del Consejo de Ministros (PCM), realizándose mesas redondas donde los investigadores mostraron los hallazgos de sus investigaciones realizadas en el contexto de la pandemia ${ }^{(5)}$.

Este número especial de SARS-CoV-2, COVID-19 y Pandemia, visibilizará investigaciones realizadas en prevención, factores de riesgo, desabastecimiento, uso y efectos adversos de medicamentos, así como sus efectos en mortalidad, calidad de vida, impacto en la salud mental y rehabilitación; así como su posible impacto en el medio ambiente.

Impulsar la investigación sobre emergencias de forma continua y sistemática, y no solo cuando ocupan los titulares y provocan el pánico. Además de respuestas rápidas a los problemas emergentes, es necesario invertir en investigación interdisciplinaria sobre problemas prioritarios de salud pública que no atraen la atención de los líderes políticos locales o globales porque son endémicos ${ }^{(6)}$. Hasta ahora, ha habido poca evidencia de coordinación internacional, a nivel mundial o dentro de bloques regionales $^{(7)}$.

El pasado, presente y futuro de la investigación, estará marcada durante muchos años por la pandemia del COVID-19, es un antes y un después en todas las áreas del saber, ya

\section{FILIACIÓN \\ 1. Presidente Ejecutivo, Seguro Social del Perú, EsSalud, Lima, Perú. \\ 2. Universidad Nacional Mayor de San Marcos, Lima, Perú. \\ a. Doctor en Farmacia y Bioquímica. \\ b. Investigador RENACYT. \\ ORCID: \\ 1. Mario Carhuapoma Yance 10000-0003-4669-6384 \\ CORRESPONDENCIA \\ Mario Carhuapoma Yance \\ EMAIL \\ mcarhuapmay@unmsm.edu.pe}

\section{CONFLICTOS DE INTERÉS}

El autor, niega conflictos de interés

\section{COMO CITAR}

Carhuapoma-Yance, M. (2021). Desafíos de la pandemia COVID-19: Investigación en salud y su divulgación. Revista Del Cuerpo Médico Hospital Nacional Almanzor Aguinaga Asenjo, 14(Sup1), 6 - 7. https://doi.org/10.35434/rcmhnaaa. 2021.14Sup1.1139 
que la afectación no está circunscrita solo a la salud(8). Necesitamos respuestas unificadas a las pandemias en lugar de diversas estrategias desconectadas, y realizar investigación multidisciplinaria son parte del desafío en el Perú.

\section{REFERENCIAS BIBLIOGRÁFICAS}

1. Bravo-García E, Magis-Rodríguez C. La respuesta mundial a la epidemia del COVID-19: los primeros tres meses. Bol Sobre COVID-19 Salud Pública Epidemiol Dep Salud Pública Fac Med UNAM Rev En Internet. 2020;1(1):1-8.

2. Respuestas al COVID-19 desde la ciencia, la innovación y el desarrollo productivo | Publications [Internet]. [citado 28 de septiembre de $\left.\begin{array}{lllll}2 & 0 & 2 & 1\end{array}\right] . \quad D$ is p o n i b l e e n : https://publications.iadb.org/publications/spanish/document/R es puestas-al-COVID-19-desde-la-ciencia-la-innovacion-y-el- desarrollo-productivo.pdf

3. Vásquez-Uriarte K, Roque-Henriquez JC, Angulo-Bazán Y, Ninatanta Ortiz JA. Análisis bibliométrico de la producción científica peruana sobre la COVID-19. Rev Peru Med Exp Salud Pública. 2021;38:224-31.

4. Pese a crisis, Latinoamérica financia investigación en COVID-19 [Internet]. América Latina y el Caribe. [citado 28 de septiembre de 2021]. Disponible en: https://www.scidev.net/americalatina/news/pese-a-crisis-latinoamerica-financia-investigacion-encovid-19/

5. INS realizará Primer Congreso Científico COVID-19 a fin de promover la investigación en el país [Internet]. [citado 28 de septiembre de $\left.2 \begin{array}{llll}2 & 0 & 2 & 1\end{array}\right]$

D i s p o n i ble

e $n$ : https://www.gob.pe/institucion/minsa/noticias/524062-insrealizara-primer-congreso-cientifico-covid-19-a-fin-de-promoverla-investigacion-en-el-pais

6. Ventura D de FL, Ribeiro H, Giulio GM di, Jaime PC, Nunes J, Bógus CM, et al. Desafios da pandemia de COVID-19: por uma agenda brasileira de pesquisa em saúde global e sustentabilidade. Cad Saúde Pública. 2020;36:e00040620. 